Response to "Orthopaedics and industry: an uneasy alliance?" Dear Sir,

I read with interest the article by Field, Shimmin and Cattani' on the socalled uneasy alliance between orthopaedics and industry.

The times have certainly changed over the past twenty years in terms of the relationship, rules and procedures between Health Care Providers and Industry. It is in the perspective of the individual to decide if these changes have been for the better or the worse. Personally I can make an argument in either direction depending on the specific topic. For instance, it never ceased to amaze me that I could walk in the front door of any hospital in the US, find my way to the female locker room, change into scrubs and be inside an OR within 15 minutes without anyone asking me for identification. Today, the US vendor certification process has attempted to provide a credentialing service to document who should and should not be in critical areas. This is a positive step to protect not only patient confidentiality but hospital staff safety. But do we really need to wear bright red hats and paper jump suits to let everyone know that "industry is in the house"?

How have these changes impacted product innovation? Years ago contracts were not required for consulting services. As a design engineer I was free to schedule a cadaver lab with a surgeon and "invent". Many times, however, this development session ended up as a cosy rendezvous between only myself and the cadaver as the surgeon cancelled at the last minute. The surgeons were doing this free of charge and paying patients had precedence. Today surgeon consulting agreements provide clarity into the terms of the relationship, fair market value payment rates, topics covered by the agreement, etc. The consultancy payment for hours/travel etc., has improved the consistency of the sessions. In addition the ownership of any resulting design element is documented before the session so there is clarity before any designing is initiated. However, the movement towards billable hours for consultancy services has reduced the opportunity for impromptu conversations or a quick question through email. Now, for some, these conversations are billed for by the surgeon consultant and must be documented by the person from industry in order to support payment.

One thing that has not changed throughout all of this is the need for the relationship between industry and the surgeon in order to bring technology forward and benefit the patient, which should always be the end goal. I hope we never see the day when engineers are prohibited from live surgical observation due to tightening regulations, as a bioskills lab cannot fully simulate the environment and challenges that a surgeon is faced with, and in my opinion, will hamper innovation.

\section{Lisa Donnelly,}

Boston, Massachusetts

\section{REFERENCES}

1. Field R, Shimmin A, Cattani L. Orthopaedics and industry: an uneasy alliance? Bone \& Joint 360 2012;1(2):7-10. 